\title{
Diminutivos, hipocorísticos y otras formas de nombrar a los parientes en familias de Santiago de Chile $^{1}$
}

\author{
Diminutives, hypocoristics and other ways of naming \\ relatives in families in Santiago de Chile
}

\author{
Herminia Gonzálvez \\ Universidad Central de Chile \\ herminiagonzalvez@gmail.com \\ Santiago de Chile
}

\section{RESUMEN}

En este artículo se exponen resultados procedentes de una investigación antropológica titulada "Las familias en Chile: el trabajo de parentesco y la generación de constelaciones familiares". En concreto, en este trabajo se trata de dar respuesta a uno de los objetivos específicos de la investigación señalada, el cual consiste en mostrar cómo el trabajo de parentesco se relaciona con la forma de nombrar a los parientes. Para ello partimos de la definición de trabajo de parentesco de Di Leonardo definida como "la concepción, el mantenimiento y las celebraciones rituales a través de los lazos de parentesco dentro del grupo doméstico, incluyendo visitas, cartas, llamadas telefónicas, regalos y tarjetas recordatorias; la organización de las reuniones por vacaciones (...)" (1987: 442), para indagar cómo este trabajo repercute en las formas de nombrar. La metodología utilizada han sido las entrevistas en profundidad las cuales han permitido construir las cartas de parentesco de cada uno de los entrevistados/as. Como resultados encontramos que el uso de los diminutivos, los hipocorísticos y otras formas de nombrar son expresiones de ese trabajo de mantenimiento de los vínculos que se produce al interior de las familias el cual se encuentra influenciado por las posiciones de género y parentesco y las formas familiares que ocupan las personas entrevistadas.

Palabras clave: Trabajo de parentesco; Formas de nombrar; Familias; Género; Diminutivos; Hipocorísticos.

\section{SUMMARY}

This article explains the results of an anthropological investigation entitled "Families in Chile: Kinwork and the Generation of Family Constellations", and seeks to tackle one of the specific research goals: to show how kin work affects the naming of relatives. To do so, we use Di Leonardo's definition of kin work "the conception, maintenance, and ritual celebration of crosshousehold kin ties, including visits, letters, telephone calls, presents, and cards to kin; the or-

\footnotetext{
${ }^{1}$ La autora agradece a la Comisión Nacional de Investigación Científica y Tecnológica de Chile (CONICYT), que financia el presente estudio a través del proyecto FONDECYT 11121245 "Las familias en Chile: el trabajo de parentesco y la generación de constelaciones familiares" que ella dirige. Asimismo, la autora agradece los comentarios realizados por Francisca Ortíz y Sofía Larrázabal a este trabajo.
} 
ganization of holiday gatherings (...)" (1987: 442) to investigate its impact on ways of naming relatives. The method used was in-depth interviews, which allowed us to construct kinship charts for each respondent. We found that the use of diminutives, hypocoristics and other ways of naming express the maintenance of kinship ties that are produced inside families. In turn, these ways of naming are influenced by gender and kinship positions and by the positions that respondents use to identify themselves inside their families.

Key words: Kin Work; Ways of Naming; Families; Gender; Diminutive; Hypocoristic.

\section{INTRODUCCIÓN: RELEVANCIA DEL TEMA, ESTADO DE LA CUESTIÓN Y PRINCI- PALES HIPÓTESIS}

En este artículo se exponen resultados procedentes de una investigación antropológica titulada "Las familias en Chile: el trabajo de parentesco y la generación de constelaciones familiares". Esta investigación que tuvo por objetivo general analizar el trabajo de parentesco que realizan hombres y mujeres para reorganizar sus vínculos familiares y de parentesco y gestionar los procesos de tensión a los que la sociedad les somete, se apoya en la articulación del género y el parentesco como enfoques analíticos indisociables (Collier y Yanagisako 1987; Gregorio 2011; Del Valle 2010; Gregorio y Gonzálvez 2012) para el análisis de las familias en Santiago de Chile. Específicamente, este artículo da respuesta a uno de los objetivos específicos de esta investigación, que consiste en mostrar cómo el trabajo de parentesco se relaciona con la forma de nombrar a los parientes. Para ello partimos de la definición de Di Leonardo quien originalmente conceptualizó este trabajo como "la concepción, el mantenimiento y las celebraciones rituales a través de los lazos de parentesco dentro del grupo doméstico, incluyendo visitas, cartas, llamadas telefónicas, regalos y tarjetas recordatorias; la organización de las reuniones por vacaciones (...)" (1987: 442). Con esta noción Di Leonardo (1987) visibilizó aquellas prácticas sociales acometidas principalmente por las mujeres, trabajos que producían parentesco e identidad colectiva, pero que no eran considerados como trabajo por la naturalización que comportaba su asociación con lo femenino y lo doméstico (Gregorio y Gonzálvez 2012). Este concepto, trabajo de parentesco (Di Leonardo 1987), acuñado hace más de dos décadas ${ }^{2}$, ha sido poco utilizado como tal en los trabajos actuales sobre familia en Chile (Gonzálvez 2013, 2016). A partir de esta categoría analítica se realiza una aproximación al análisis de las familias residentes en Santiago de Chile, desde la indagación de las responsabilidades y prácticas sociales que se derivan del trabajo de mantenimiento de los vínculos en relación con las formas de nombrar a los parientes.

Así, la noción de familia que orienta este estudio es aquella que las considera como unidades sociales complejas, de amplia diversidad estructural, cultural, económica, cuyos individuos en relación cumplen funciones sexuales, económicas, reproductivas, socializadoras, presentando en la práctica tal variabilidad, que no siempre es fácil clasificarlas (Alberdi 1999; Jelin 1998; Rivas 2007; Yanagisako 1979). La noción de parentesco remite a la forma en que los diferentes grupos humanos se representan y teori-

${ }^{2}$ Es importante mencionar que este concepto ha sido utilizado posteriormente a Di Leonardo (1987) en los trabajos de Carrington (1999). 
zan los procesos de procreación, concepción y gestación, así como al papel sociosubjetivo de los sexos en tales procesos (Tubert 2001).

A partir de lo mencionado, en esta investigación se postula que las familias, a través del trabajo de parentesco, reaccionan frente al entorno y no lo hacen de forma única, persistente, ni lineal, sino que sus formas de acción repercuten en diversos campos, como la organización del espacio (residencia) y del tiempo (las memorias), las representaciones de género, el mantenimiento de los vínculos familiares y de parentesco, la forma de nombrar a los parientes y la manera de definir las reciprocidades entre ellos. Tales renovaciones pueden ser más o menos duraderas y pueden representar innovaciones o regresiones (crisis, rupturas, entre otras) (Gonzálvez, 2016). Esto es así, porque las familias son, por un lado, procesos dinámicos, de creación, mantenimiento y actualización de vínculos familiares y de parentesco pero, por otro lado, son un trabajo, en tanto requieren un esfuerzo constante por mantener conectadas a las personas que se consideran parte de la relación. En particular, en este artículo nos centramos en cómo el trabajo de parentesco se relaciona con la forma de nombrar a los parientes observando las responsabilidades que existen bajo las posiciones de género y parentesco de los/as entrevistados/as (madre, padre, hijo, entre otras) — términos de referencia-, pero analizando los usos y manipulaciones que se esconden detrás de los términos de apelación (Luchito, Ale, entre otras formas de nombrar). Es decir, que el uso de los términos de parentesco que utilizan los miembros de la familia es una dimensión más de análisis del trabajo de parentesco que permite explicar, cómo desde las formas de nombrar a los parientes se crean posiciones de poder diferenciadas al interior de las familias, en tanto nombrar también contribuye a la naturalización de determinadas prácticas de mantenimiento de los vínculos. Es más, estas formas de nombrar pueden a su vez expresar relaciones pasadas y/o actuales. Se postula que esto es así, porque las posiciones de género y parentesco que ocupan cada uno de los miembros de la unidad familiar y las prácticas sociales que se derivan de estas posiciones influyen en las formas de ser nombrados tanto en el pasado como en el presente.

Para ello, partimos de la premisa que señala que:

Nombrar es asignar y reconocer una posición y un rol determinados a aquella persona de la que hablamos (términos de referencia) y/o a la que nos dirigimos (términos de apelación); implica situar a alguien en un espacio genealógico y social, del que se derivan actitudes, comportamientos, prácticas, sentimientos, derechos y deberes, expectativas recíprocas, legitimadas socialmente por los modelos de parentesco dominantes en cada sociedad. Nombrar es dotar de identidad a una persona, ubicarla en el sistema de representación de nuestros parientes, los que son y los que no son de los "nuestros". Los referentes que dotan de significado a los términos de parentesco son las categorías y grupos de parentesco que predominan en una sociedad dada (Moncó y Rivas 2007:1).

Además, Segalen señala que las manipulaciones individuales de los términos de parentesco revelan el sutil juego de las relaciones sociales (2007:44). Producto de esta premisa (Rivas y Moncó 2007), nuestra hipótesis de partida señala que la forma de nombrar a los parientes, pero sobre todo, la forma de "manipular" los términos relativos al parentesco expresan la manera en la cual los miembros de las familias intencionan el tipo de trabajo de mantenimiento de los vínculos que se quiere lograr al interior del grupo familiar, en definitiva, los términos que se utilizan orientan el trabajo de parentesco. 
En consecuencia, la pregunta que subyace a esta hipótesis es ¿cómo el uso de los términos de parentesco por parte de las familias en el contexto chileno, en concreto, Santiago de Chile, produce y mantiene diferencias de género? ¿Cómo mostrar esas diferencias desde la investigación social? Para tratar de responder a las preguntas formuladas, hemos realizado 30 entrevistas producto de las cuales se realizaron 30 cartas de parentesco (Comas d'Argemir 2010), técnica que permite analizar la organización social de la vida familiar a partir de la posición de parentesco de la persona entrevistada.

\section{PARENTESCO, GÉNERO Y DIVERSIDAD FAMILIAR: LAS FORMAS DE NOMBRAR}

Hasta los años 80 del siglo XX, el concepto de parentesco que predominó en la Antropología fue aquel caracterizado por la prevalencia del énfasis de los vínculos biológicos (Scheneider 1968, 1984; Segalen 1992), donde la familia era entendida desde el modelo biologicista, en el que la sexualidad reproductiva se comprendía dentro de los límites que imponía el matrimonio. Las críticas realizadas al mencionado "modelo estándar del parentesco" (Needham, 1971; Schneider 1984; Grau 2006) dieron paso al "modelo constructivista del parentesco", el cual, en palabras de Bestard, se diferencia del primero

en la medida en que extiende a la consanguinidad y, en particular, a la filiación, el estatus de construido socialmente que el modelo estándar reservaba a la afinidad. Si este modelo corresponde a lo que en la teoría de la alianza denominamos estructuras complejas, el modelo constructivista correspondería a lo que podríamos denominar un parentesco postcomplejo (2009:89).

El hecho de que autores como Scheinder (1984) desnaturalizaran el estudio del parentesco contribuyó a que la antropología feminista pudiera mostrar lo indefendible que era pensar en los "hechos naturales" del sexo, la procreación y la crianza de los hijos como criterios universales, revitalizando con ello el estudio del parentesco y señalando la articulación del género con esta categoría (Collier y Yanagisako 1987). El parentesco concebido como un conjunto de prácticas que ordenaban la sexualidad, el matrimonio y la filiación le permitió a la antropología feminista mostrar cómo determinados procesos, entre ellos la naturalización, transformaban las diferencias en desigualdad (Di Leonardo 1991; Lamphere 1987; Yanagisako y Delaney 1995; Gregorio 2006).

Desde esta mirada crítica del estudio del parentesco enfocada en las experiencias, comprensiones y representaciones cotidianas de género, poder y diferencia, la familia dejó de conceptualizarse como una unidad armoniosa para pasar a ser analizada como un sistema de relaciones de poder (León 1994: 36), es decir, como

una organización social, un microcosmos de relaciones de producción, de reproducción y de distribución, con una estructura de poder y con fuertes componentes ideológicos y afectivos que cementan esa organización y ayudan a su persistencia y reproducción. Dentro de ella también se ubican las bases estructurales del conflicto y la lucha, ya que al tiempo que existen tareas e intereses colectivos o grupales, los miembros tienen deseos e intereses propios anclados en su propia ubicación dentro de la estructura social (Jelin 1998:26). 
Así, las críticas incorporadas a los estudios del parentesco no sólo desde el feminismo sino también desde el marxismo o la historia significaron el paso de una teoría del parentesco donde el análisis de las relaciones de género —y de poder- estaban ausentes, a la necesidad de incorporarlas. En definitiva, el género, como también lo hiciera la salud, o el cuerpo, transformaron el estudio del parentesco al dotar al individuo de agencia, es decir, al convertirlo en un agente activo con iniciativa propia.

A partir de entonces el ámbito del parentesco en general, y de la institución familiar en particular se han caracterizado por la importancia y la radicalidad de las transformaciones experimentadas en las últimas tres décadas (Rivas 2007; Gonzálvez 2013). Nos referimos a opciones que "van más allá de la sangre". Hoy sabemos que los límites entre la biología y la elección son cada vez más difusos (Rivas 2009; Bestard 2009; Sanz et al. 2013), aumentando los trabajos de investigación que incorporan el estudio de las múltiples expresiones del parentesco (Scheffler 1991; Carsten 2004; Yanagisako 1979; Peletz 1995; Yanagisako y Delaney 1995). En consecuencia, cada vez son más los/as estudiosos/as de las ciencias sociales que se centran en el análisis de las relaciones de parentesco desde la diversidad de formas familiares existentes (Arriagada 2008; Bestard 2009; Robichoux 2007; Cadoret 2003; Rotenberg y Agress Wainer 2007; Jociles y Rivas 2010; Jociles y Medina 2013; González 2009; Gonzálvez 2013, 2015; Gregorio y Gonzálvez 2012; Olavarría 2014; Salvo 2015; Sanz et al 2013; Tasker 2005) desencadenando oportunas formas de comprender las relaciones familiares y de parentesco que no se centran únicamente en los hechos de la reproducción, la biología o la genealogía para establecer quiénes son considerados parientes y quienes no, sino que también prestan atención a las múltiples opciones de sentir, construir y practicar la familia (Konvalinka 2012). Desafíos para la investigación social, que en palabras de Rivas son producto, por un lado, de las elecciones, estrategias y prácticas de los actores quienes van construyendo múltiples maneras de emparentamiento en las que la biología, la elección y la voluntad están no en relación de oposición, sustitución, asimilación, y/o sucesión, sino en relación de adición, complementariedad, acumulación y/o sucesión (Rivas 2009: 15). Y por otro lado, consecuencia de los efectos que los parentescos electivos y las plurales modalidades de emparentar provocan en las instituciones que intervienen en los procesos de decisión y legislación sobre los asuntos de parentesco (Rivas 2009: 15).

\section{LAS FORMAS DE NOMBRAR EN LA ACTUALIDAD}

Cuando una persona se dirige a otra considerada como pariente, puede hacerlo usando el nombre propio o bien un término que especifica la relación de parentesco existente $^{3}$. Si bien, han sido muchos los esfuerzos empleados en el estudio y análisis de los términos de parentesco, lo que la terminología explica permite describir y com-

\footnotetext{
${ }^{3}$ Palerm comenta que el primer paso fundamental para el estudio de las terminologías de parentesco fue dado por Morgan (1871) a partir de su obra "Sistemas de consanguinidad y de afinidad". (...) Después de Morgan, Kroeber y Lowie, fueron quienes aplicaron una serie de criterios de clasificación y análisis de tecnonimias, (aproximadamente nueve), sin embargo, no constituyeron todavía un verdadero sistema de clasificación de las terminologías (Palerm 1997:153).
} 
prender la estructura y funcionamiento de una sociedad, sacando conclusiones sobre los procesos de cambio social (Palerm 1997:154).

Como se comentara al inicio de este artículo, el uso de los términos de parentesco en las familias en Santiago de Chile es parte del cometido de este trabajo, desde el entendido de que la forma de nombrar opera tanto para las formas familiares fuertemente institucionalizadas, es decir, aquellas en sintonía con el modelo bio-conyugal y para las familias menos institucionalizadas, es decir, aquellas donde los límites entre elección y la biología se difuminan. Si bien en Chile el estudio de las formas de nombrar es algo todavía reciente, aquellas desafiadas por la diversidad familiar todavía se encuentran más alejadas de los análisis. Los retos que impone a las formas de nombrar los estudios sobre la homoparentalidad, la monoparentalidad por elección, las familias transnacionales y las familias recompuestas son algunas de las emergencias en relación con la diversidad familiar que van apareciendo como "nuevos" fenómenos sociales a analizar (algunos no tan nuevos). Por ejemplo, en los trabajos sobre la monoparentalidad por elección, (Jociles y Rivas 2010; Jociles y Villaamil 2012; Jociles y Medina 2013; Salvo y Gonzálvez 2015; Jociles y Medina 2013) en el caso de la adopción, se multiplican los términos de parentesco en tanto puede suceder que la madre adoptiva sustituya a la madre biológica — aunque no siempre es así-. Sin embargo, en el caso de la reproducción asistida, nadie sustituye a nadie, ya que nos encontramos que existen los donantes de esperma o de óvulos (madres y /o padres genéticos), la madre social (la personas o personas que inician el proceso porque quieren ser padres), y la mujer que recibe el óvulo fecundado (madre biológica). La madre biológica puede coincidir con la madre social, pero no con la genética (Rivas 2009: 14). Esta multiplicidad de conceptos también la vemos en el caso de las familias reconstituidas donde las relaciones conyugales y las relaciones filiales se disocian (Moncó y Rivas 2007), o en los casos de las familias transnacionales (Gregorio y Gonzálvez 2012; Gonzálvez 2013; Gonzálvez 2016) donde sucede que quienes ocupan determinadas posiciones, por ejemplo, la de abuela, termina cumpliendo funciones asignadas a la madre con aquellos nietos/as que se quedaron a su cuidado en origen. En estos casos, los nietos/as ven a sus abuelas como madres, generando formas de nombrar que adquieren un sentido práctico.

En relación con lo anterior,

necesitamos finos análisis de situaciones familiares en las cuales el tema de interés sea el nuevo vocabulario del parentesco que surge en situaciones fluidas no esposo, medio hermano o pseudo hermano; medio abuela, etc. y a las relaciones que se forjan en el interior de estas nuevas configuraciones de parentesco basadas, ya no en el lazo biológico, sino en el lazo social (Le Gall y Martin 1988 en Segalen 2007: 54).

En definitiva, se necesitan trabajos que permitan explicar desde los términos de parentesco las relaciones sociales que se suceden al interior de las familias, sean estas formas familiares sustentadas en lazos consanguíneos o no.

\section{METODOLOGÍA: ENTREVISTAS EN PROFUNDIDAD Y CARTAS DE PARENTESCO}

El cumplimiento del objetivo que se responde en este artículo, es decir, indagar cómo la forma de nombrar a los parientes, pero sobre todo, la forma de "manipular" 
los términos relativos al parentesco, se relaciona con la manera en la cual los miembros de las familias mantienen sus vínculos, implicó la realización de la "carta de parentesco" de las 30 personas entrevistadas. Las cartas de parentesco son

el resultado de la reconstrucción de los parientes a partir de un ego (que es como se llama a la persona que se toma como punto de referencia), más allá de quienes comparten su grupo de residencia. Las relaciones de parentesco que se presentan en las cartas de parentesco son, de hecho, una clase de red social, una parte del conjunto de relaciones que tiene un individuo (Comas d'Argemir 2010:145).

La carta de parentesco, aunque parte de un ego, como también lo hace el árbol genealógico, facilita la incorporación de personas consideradas parientes compartan o no vínculos biológicos. Para construir la carta de parentesco se trató, por medio de la entrevista en profundidad, de recomponer el universo de las relaciones de parentesco de cada entrevistado/a y, al mismo tiempo, obtener información sobre las relaciones y prácticas sociales relacionadas con el mantenimiento de los vínculos familiares que mantienen con sus parientes y la percepción que el entrevistado/a tiene de estas relaciones.

La carta de parentesco se realizó por medio de la entrevista semiestructurada la cual se inició preguntando por los diversos miembros del grupo residencial y por los parientes que reconocen como tales (vivieran juntos o no) en el momento de la entrevista, incluyendo sus datos personales y relación de parentesco, así como la frecuencia en la comunicación y contacto entre ellos, y las formas de colaboración económica, afectiva, asistencial o de otro tipo existente entre cada pariente. También se les hizo esta misma pregunta respecto de estas mismas relaciones años atrás, y de aquellas otras relaciones que ellos quisieron mencionar libremente, para construir una carta de parentesco donde se pudiera contrastar los elementos de la diacronía con la sincronía. En relación con esto último se recogieron durante la entrevista los términos de parentesco utilizados en cada relación familiar para hacer un "Repertorio" de los términos de parentesco utilizados en las entrevistas de la muestra.

Se utilizó la carta de parentesco porque, a diferencia del árbol genealógico, permite conocer no sólo "al conjunto de relaciones genealógicas mantenidas en marcha, llamadas aquí parentesco práctico, sino también al conjunto de las relaciones no genealógicas que pueden ser movilizadas para las necesidades ordinarias de la existencia, llamadas aquí relaciones prácticas" (Bourdieu 2008: 265). Con la carta de parentesco se analiza el "parentesco oficial definido de una vez y para siempre por las normas protocolares de la genealogía, y el parentesco práctico, cuyas fronteras y definiciones son tan numerosas y varias como los usuarios y las ocasiones de ser utilizados. Es el parentesco práctico el que configura los matrimonios; es el parentesco oficial el que los celebra" (Bourdieu, 2008:267). De esta forma, el parentesco, en esta investigación es considerado como una "construcción social", que implica, en palabras de Bourdieu que

las relaciones de parentesco son algo que se hace y con las que se hace algo, no es solamente, como podrían hacerlo creer las taxonomías en vigor, sustituir una interpretación "estructuralista" por una interpretación "funcionalista"; es cuestionar radicalmente la teoría implícita de la práctica que lleva a la tradición etnológica a aprehender las relaciones de parentesco "bajo la forma de objeto o de intuición", como advierte Marx, en lugar de bajo 
la forma de las prácticas que las producen, las reproducen o las utilizan con referencia a funciones necesariamente prácticas (Bourdieu, 2008:265).

En el desarrollo de la carta de parentesco se consideró no sólo la composición del hogar de la persona entrevistada y quiénes consideran que son sus parientes, sino también, el parentesco desde el cual se definían (padre, madre, hermana, hija, amiga), y los usos y funciones de ese parentesco en relación con las personas que incluían como parte de su familia. A continuación se presenta un ejemplo de una carta de parentesco de la entrevistada Mujer_8_2013:

\begin{tabular}{|c|c|c|c|c|c|c|c|}
\hline N. ${ }^{\circ}$ & \begin{tabular}{|l|} 
FORMA FAMIIIAR \\
NUCLEAR BIPA- \\
RENTAL CON HIJOS \\
FUERA DEL HOGAR \\
(MUJER_8_2013)
\end{tabular} & EDAD & $\begin{array}{l}\text { Trabajo/ } \\
\text { JORNADA }\end{array}$ & $\begin{array}{l}\text { LUGAR DE } \\
\text { NACIMIENTO }\end{array}$ & \begin{tabular}{|l} 
LUGAR DE \\
RESIDENCIA/ \\
COMUNA
\end{tabular} & \begin{tabular}{|l} 
TÉRMINOS \\
DE REFERENCIAS \\
EN RELACIÓN \\
CON EGO
\end{tabular} & \begin{tabular}{|l} 
TÉRMINOS \\
DE APELACIÓN \\
EN RELACIÓN \\
CON EGO
\end{tabular} \\
\hline 1 & Vivian Gavilán & 54 & Dueña de casa & $\begin{array}{l}\text { Comuna San } \\
\text { Miguel, Región } \\
\text { Metropolitana }\end{array}$ & Peñalolén & $E G O$ & \\
\hline 2 & Mario & 56 & Psicólogo & $\begin{array}{l}\text { Curicó, Región } \\
\text { del Maule }\end{array}$ & Peñalolén & Esposo & Marito \\
\hline 3 & Mauricio & 30 & Psicólogo & $\left|\begin{array}{l}\text { Ñuñoa, Región } \\
\text { Metropolitana }\end{array}\right|$ & Ñuñoa & Hijo & Mauri \\
\hline 4 & Claudia & 28 & Actriz & $\begin{array}{l}\text { Peñalolén, } \\
\text { Región } \\
\text { Metropolitana }\end{array}$ & Ñuñoa & Hija & Claudia \\
\hline 5 & Katina & 26 & Estudiante & $\begin{array}{l}\text { Peñalolén, } \\
\text { Región } \\
\text { Metropolitana }\end{array}$ & La Florida & Hija & Katina \\
\hline 6 & Mamá & 77 & Dueña de casa & $\begin{array}{l}\text { San Carlos, } \\
\text { Región del } \\
\text { Bíobío }\end{array}$ & Macul & Mamá & Mamá \\
\hline 7 & Vicente & 6 & Estudiante & $\begin{array}{l}\text { Peñalolén, } \\
\text { Región } \\
\text { Metropolitana }\end{array}$ & Ñuñoa & Nieto & $\begin{array}{l}\text { Vicentito/ } \\
\text { Pochoquito }\end{array}$ \\
\hline \multicolumn{8}{|c|}{ FAMILIA EXTENSA } \\
\hline 8 & Hernán & 40 & Empresario & $\left|\begin{array}{l}\text { Ñuñoa, Región } \\
\text { Metropolitana }\end{array}\right|$ & Peñalolén & Hermano & Hernán \\
\hline 9 & Nathalie & 45 & Odontóloga & $\begin{array}{l}\text { Ñuñoa, Región } \\
\text { Metropolitana }\end{array}$ & Temuco & Hermana & Naty \\
\hline 10 & Carlos & 61 & Vendedor & $\begin{array}{l}\text { Curicó, Región } \\
\text { del Maule }\end{array}$ & Gran Avenida & Cuñado & Carlos \\
\hline 11 & Luis & 68 & Taxista & $\begin{array}{l}\text { Curicó, Región } \\
\text { del Maule }\end{array}$ & Curicó & Cuñado & Lucho \\
\hline 12 & Ana & 66 & Contadora & $\begin{array}{l}\text { Curicó, Región } \\
\text { del Maule }\end{array}$ & Curicó & Cuñada & Negra \\
\hline
\end{tabular}


Derivado de estos usos, las prácticas de mantenimiento de los vínculos sobre las que se conversó en las entrevistas y que componen ese trabajo de parentesco fueron: celebraciones no religiosas, tradiciones y costumbres, prácticas religiosas, visitas, llamadas telefónicas, envío de cartas, emails, uso de Twiter, Facebook o chat, videoconferencias, envío o recepción de regalos, entrega de tarjetas de recuerdo, préstamos de dinero, remesas económicas (migrantes), dar cuidados, y recibir cuidados, acontecimientos familiares y otras formas de mantenimiento de los vínculos.

\section{POSICIONES DE PARENTESCO Y FORMAS FAMILIARES}

En relación al análisis de las entrevistas encontramos que desde el parentesco, los entrevistados/as se definían desde el lugar de padres, madres, abuelas, hijos/as, incluso, amigos/as, y hermanos/as, siendo estos parentescos los que mantienen activos los vínculos con aquellas personas consideradas de la familia, o "casi" de la familia (como mencionan algunos de los entrevistados/as). No apareció en la muestra hombres que se identificaran con la posición de parentesco "abuelos" o "tíos". Las mujeres son quienes representan más parentescos, sobre todo, el parentesco "madre" e "hija", sin obviar que fueron más mujeres entrevistadas que hombres. Asimismo predominó el género femenino en la muestra reflejándose más las prácticas familiares de mujeres (24 entrevistadas) que de hombres ( 6 entrevistados). Específicamente, las formas familiares entrevistadas producto del cruce entre el ciclo de vida ${ }^{4}$ y la clase social ${ }^{5}$ fueron: unipersonal, extensa biparental, extensa monoparental, familia biparental sin hijos, familia nuclear con hijos en el hogar y con hijos fuera del hogar, familia nuclear monoparental y familia reconstituida. Derivado de ello, las formas familiares se constituyeron como puntos de partida tratando de superar una de las características predominantes en los estudios sobre familia, la cual implica acercarse al análisis de la misma a través del hogar ${ }^{6}$. Aunque se parte de la composición del hogar para definir las formas familiares, tratamos de superar esta mirada que establece como sinónimo familia y hogar a partir del concepto de forma familiar el cual acoge aquellas relaciones que van más allá de las personas que viven juntas. Así, Cicerchia (1999) propone que cuando se haga referencia a la familia se emplee el concepto de "formas familia-

\footnotetext{
${ }^{4}$ En cuanto al ciclo de vida, se entrevistaron solteros, parejas sin hijos, parejas con hijos, y parejas con hijos adultos fuera del hogar.

5 Jelin menciona que «la variable clase o estrato social, se alza como una variable significativa en la medida que incide de manera importante en los márgenes de libertad de acción y decisión por parte de las mujeres. Consecuentemente define variaciones en las relaciones de género (1995: 407).

${ }^{6}$ En este sentido, Valenzuela, Tironi y Scully cuentan que "la ventaja de pensar la familia en torno a las personas que viven juntas en un hogar es que la hace fácil de observar. Por lo mismo, gran cantidad de investigadores e instituciones optan por trabajar con esta definición operativa" (2006: 19) sin embargo, los autores reconocen las deficiencias de este enfoque sobre el cual se sitúan señalando que "la dimensión familiar de las personas que viven solas, se pierde con esta definición práctica, así como el vínculo también permanente aunque variablemente importante entre los hijos adultos y los padres" (2006: 19). En efecto, reconocer esta limitación para este trabajo implica mirar más allá de los datos registrados en los censos, ya que los datos nos explican poco sobre las circunstancias cotidianas de las familias, por ejemplo, la relación entre los padres y sus hijos adultos o en relación a la familia extensa.
} 
res" dado que se trata de una organización que como sujeto histórico complejo es receptora de cambios y de determinantes sociales. Adoptar dicho concepto permite dar voz a la perspectiva de los sujetos entrevistados y aproximarnos a la definición de familia con la que ellos se identifican, agrupando de esta forma una amplia diversidad de acuerdos familiares. En concreto, las formas familiares y las posiciones de parentesco con las que se identifican los entrevistados/as son las siguientes:

1) Once fueron las personas entrevistadas que son parte de "formas familiares unipersonales" ${ }^{7}$. En estos casos se definen sobre todo desde la posición de madre o hijos/as, predominando el vínculo materno-filial. En estos ejemplos es claro que la composición del hogar no dice relación con las personas que se mencionan como parte de la familia, incluyendo a aquellas con quiénes mantienen contacto, aunque éstas estén en otros hogares, e incluso países ${ }^{8}$. Quienes se identifican principalmente como "madres" incluyen a sus hijos, nueras/yernos, y nietas como parte de su familia, aquellos que se identifican como "hijos/as", incluyen a sus padres (padre y madre), pero también a otros miembros del núcleo familiar como son las primas/os ${ }^{9}$, tíos y amigas $^{10}$.

2) Tres fueron las personas entrevistadas que son parte de la "forma familiar extensa biparental" ${ }^{11}$. La primera de ellas se define como "esposa", "madre", "hermana" y "tía"; la segunda como "madre" y "nieta", y la tercera como "madre". Todas ellas incluyeron a muchas personas como parte de la familia, y se definieron no sólo a partir del vínculo materno-filial, sino también, a través del vínculo abuela-nieta, tía-sobrina.

3) Dos fueron las personas entrevistadas que son parte de la "forma familiar extensa monoparental" ${ }^{12}$. En ambos casos se identificaron como "hijos", predominando el vínculo filial-maternal en esta definición.

4) Dos fueron las personas entrevistadas que son parte de la "forma familiar nuclear biparental sin hijos". En uno de los casos se identificó como "hija mayor" ${ }^{13}$, y en el otro principalmente como "hermano" ${ }^{14}$.

5) Cuatro fueron las personas entrevistadas en "familiares nucleares biparentales con hijos" ${ }^{15}$. La primera de ellas se identificó como madre; la segunda como madre e hija; la tercera como hija, madre, esposa y amiga; y la última de ellas se identificó como madre. Tienen en común la autodefinición desde la posición de madre.

6) Dos fueron las entrevistas en las que se identificaron con la "forma familiar nuclear biparental con hijos fuera del hogar". La primera de ellas se posiciona como "madre, esposa, dueña de casa y abuela", y la segunda de ellas como "madre" ${ }^{16}$.

\footnotetext{
${ }^{7}$ Mujer_1_2013, Hombre_2_2013, Hombre_4_2013, Mujer_6_2013, Mujer_5_2013, Mujer_25_2013, Hombre_30_2013, Hombre_31_2013, Mujer_27_2013, Mujer_18_2013, Mujer_32_2013.

${ }^{8}$ Mujer_1_2013, Hombre_2_2013, Hombre_31_2013, Mujer_31_2013.

9 Hombre_2_2103.

${ }^{10}$ Mujer_5_2013.

${ }^{11}$ Mujer_3_2013, Mujer_18_2013, Mujer_11_2013.

12 Mujer_33_2013, Hombre_11_2013.

13 Mujer_9_2013.

${ }^{14}$ Hombre_15_2013.

15 Mujer_7_2013, Mujer_8_2013, Mujer_19_2013, Mujer_17_2013, Mujer_23_2013.

16 Mujer_25_2013, Mujer_8_2013.
} 
7) Cuatro fueron los entrevistados que se identificaron con la forma "familia nuclear monoparental" ${ }^{17}$, identificándose principalmente como "madre".

8) Y por último dos fueron las personas entrevistadas que se identificaron con la "forma familiar ensamblada". La primera de ellas se posiciona como "hija" y la segunda como "padre" ${ }^{18}$.

Aunque las personas entrevistadas forman parte de formas familiares diferentes, encontramos una serie de características compartidas. En primer lugar, el predominio de la posición de parentesco "madre" en la mayoría de las entrevistadas. En segundo lugar, que la existencia de la biparentalidad implica que la mayoría de las mujeres se identifiquen con un mayor número de parentescos, a diferencia de lo que sucede con la monoparentalidad donde predomina principalmente la posición de madre. En tercer lugar, que las personas entrevistadas son parte del conjunto de cambios sociodemográficos fundamentales que en razón del alargamiento de la esperanza de vida y el envejecimiento de la población conoce la sociedad chilena, influyendo en la composición del cuadro de las relaciones de parentesco ${ }^{19}$ que se refleja en las familias de la muestra. Esto es así ya que una de los aspectos más significativos que se detecta en las familias es que en relación con el envejecimiento de la población y los tiempos de vida, predominan las dinámicas de cuidado que practican determinados familiares con respecto a sus hijos/as y/o nietos, o sus propios padres/madres. En cuarto lugar, encontramos que aunque en algunos de los entrevistados/as se muestra cierta fragilidad respecto del vínculo conyugal (madres solteras separadas o divorciadas, mujeres y hombres que se han vuelto a casar) en la mayoría de ellos las relaciones entre los parientes ascendientes, descendientes y colaterales se refuerzan, constituyéndose en vínculos donde se establecen considerables intercambios económicos, sociales, afectivos y simbólicos. Lo mencionado se relaciona con los aportes de Olavarría cuando señala que la familia conyugal en Chile, presentada como un logro durante el siglo $\mathrm{XX}$, ha entrado en procesos de contradicciones, cambios y resistencias, dentro de las cuales destacan que padre y madre buscan su sitio a partir de nuevos valores que democratizan la vida privada, las relaciones horizontales, la mayor cercanía afectiva tanto de pareja como con los hijos, la concordancia del uso del tiempo, la transformación del papel de las mujeres en la familia y la sociedad y, la limitación del poder paterno (2014: 5).

\section{NOMBRAR A LOS PARIENTES: DIMINUTIVOS, HIPOCORÍSTICOS Y «OTRAS FOR- MAS DE NOMBRAR"}

Profundizando en el análisis de los términos de parentesco se observa en las entrevistas que cuando hombres y mujeres, en sus papeles de madre/padre, hija/o o

\footnotetext{
17 Mujer_22_2013, Mujer_28_2013, Mujer_26_2013.

${ }^{18}$ Mujer_23_2013, Mujer_24_2013.

19 Según las cifras más actualizadas a la fecha, durante el año 2013, la población de adultos mayores en el país representaba el 16,7\% de la población total, englobando a 2.885.157 personas de un total de 17.273.117 habitantes en Chile (CASEN, 2013). De éstos, el 57,3\% eran mujeres. Según las estimaciones, esta cifra aumentará a 18,9\% en el 2020, (INE, 2015), acentuándose también la feminización del envejecimiento y, en consecuencia, de los cuidados en la vejez.
} 
hermano/a refieren a sus padres y madres (términos de referencia) no hay necesidad de añadido alguno, porque padre sólo hay uno y madre sólo hay una, basta con decir "mi padre" y/o "mi madre" (Moncó y Rivas, 2007). Sin embargo, entre las forma de nombrar a los padres y a las madres (términos de apelación) sí encontramos la utilización de diferentes términos "mamá", "papá", "mamita", "papito", "mami", "papi", "vieja", "viejo", "gorda", "gordo" siendo estos dos últimos casos —viejo y gordo- utilizados tanto por jóvenes como por personas más adultas. Si bien, son diferentes conceptos para nombrar a una misma persona, hablar de los padres y de las madres incluye una mayor similitud entre el término de referencia y el de apelación, a diferencia de la forma de nombrar a otros miembros de la familia, donde el término de referencia puede ser "hijo", pero el de apelación cambia generalmente al utilizar el nombre propio del mismo $^{20}$. Este último caso sucede cuando los padres y madres nombran a los hijos/as (términos de apelación) o cuando se nombran entre hermanos/as, ya que frente al hecho de que puede haber más de un hijo/a o más de un hermano/a, se recurre a los nombres propios, o a alguna variación cariñosa de los mismos.

En relación con lo anterior, las madres predominan en el uso del diminutivo "ita" "ito" a la hora de referirse a sus hijos/as, aunque no a todos/as, de forma tal que el sufijo cobra uno u otro significado a partir de la posición de parentesco de a quien nombra. Para ilustrar mejor lo que se quiere señalar, se traen algunos casos. En primer lugar, encontramos ejemplos donde las madres lo utilizan para las hijas más pequeñas o los hijos mayores. En este ejemplo, la entrevistada utiliza el diminutivo con la más pequeña de cuatro hermanos nombrándola "Laurita" ("Mujer_6_2013", "forma familiar unipersonal"), y en el segundo ejemplo lo utiliza con la más pequeña de 3 hermanos llamándola "Barbarita" (Mujer_16_2013", "forma familiar nuclear monoparental"). Por el contrario, otra de las entrevistadas lo aplica cuando quiere mencionar al mayor de sus cinco hijos "Carlitos" con quien mantiene, según sus palabras, una relación de cierta debilidad. ("Mujer_20-2013", "forma familia nuclear extensa"). Estas entrevistas tienen en común que el uso del diminutivo conlleva una relación de cierta protección, de cuidado de los otros, predominantemente enunciado por mujeres para el caso de nuestra muestra.

En relación con lo anterior, cabe subrayar que el sufijo -ito es de lejos la variante más importante en Hispanoamérica. En México, Argentina y Chile se utiliza casi exclusivamente este sufijo (Callebaut 2011: 15). Zuluaga lo ha definido como "el diminutivo fundamental de la lengua española" (1970: 24). Andrés Bello señaló que "a los diminutivos agregamos junto con la idea de pequeñez, y a veces sin ella, las ideas de cariño o compasión, más propia de los en -ito /.../. Las de compasión o cariño no son enteramente ajenas al estilo elevado y afectuoso, pero todas ellas ocurren más a menudo en el familiar y festivo" (1984:88) ${ }^{21}$. Según Amunategui, "los diminutivos es-

\footnotetext{
${ }^{20}$ Esto mismo se encuentra reflejado en el trabajo de Schneider y Homans (19551) para estos mismos términos en relación al trabajo que realizaron con estudiantes y académicos del Departamento de Relaciones Sociales de Harvard en relación a un estudio piloto orientado a recopilar datos sobre los usos del parentesco.

${ }^{21}$ En palabras de Miguel Amunategui, Como acabo de expresarlo, don Andrés Bello no miraba con ojeriza los diminutivos. Reprobaba el abuso, pero no el uso de ellos. Se reía (según lo he oído a un testigo presencial) de que en Chile se diera diminutivo a Dios, pues algunos al despedirse solían decir adiosito y otros suplicaban por diosito; pero esto no obstaba para que Bello
} 
tán sujetos a su formación a reglas más o menos precisas, excepto los de los nombres propios, que frecuentemente son obras del capricho" (1904: 706). En las entrevistas realizadas, el sufijo se coloca en el hijo/a mayor, o en el hijo/a menor, denotando la existencia de una relación de mayor protección, cuidado o debilidad, pero no de mayor cariño con respecto a los demás hijos/as.

Pero también vemos ejemplos donde este diminutivo no se utiliza con los hijos. María, quien se identifica sobre todo como madre ("Mujer_01_2013, forma familiar unipersonal») menciona que con sus dos hijos, utiliza el nombre propio para nombrarlos, estos son "Luis" y "Carlos". El diminutivo es utilizado por ella solo para su nieta, la hija de su hijo mayor. Cuando utiliza el término de referencia acude a la palabra "hijo" y cuando utiliza el término de apelación, se mencionan los nombres. En las entrevistas también cuenta que sus hijos le dicen a ella o "madre" o "María", pero en ningún caso se utilizan estos nombres con diminutos u otra variante que denote proximidad ${ }^{22}$.

Eh, encontrarnos en mi cumpleaños es un rito que me gusta para estar con la gente que quiero. Entonces, yo lo organizo, lo hago. Eh, pero ponte tú, el cumpleaños de Carlos si él no me dice que nos veamos, me quedo triste, pero yo no tengo nada que hacer. ("Mujer_01_2013, forma familiar unipersonal")

A lo largo del análisis de las entrevistas, también encontramos los hipocorísticos como otras de las formas predominantes a la hora nombrar a los hijos/as por parte de las madres, o a las nietas/os por parte de las abuelas/os así como también en la relación entre hermanos/as. Así, los hipocorísticos serían "además de determinadas formas diminutivas, las deformaciones (generalmente por truncamiento) de nombres propios o comunes para provocar efectos de familiaridad o cariño" (Morera 2006: 206) ${ }^{23}$. Por ejemplo, en las entrevistas observamos casos donde Francisca pasa a ser nombrada como Pancha, Alejandra como Ale, o aparecen ejemplos como "Luchito" donde el hipocorístico es completado con el morfema diminutivo -ito. Lo que resulta relevante en el uso de los hipocorísiticos es que la utilización de los mismos lleva incorporada una intencionalidad en el ejercicio de las relaciones familiares. Por ejemplo, en el siguiente caso, la abuela vincula la forma de nombrar con el afecto. Ella relata lo siguiente: "mi nieta, que es como mi motivo de vida, yo le digo Lochita, pero ella se llama Eloísa"( "Mujer_19_2013, Forma familiar nuclear biparental con hijos").

Sin embargo, también encontramos "otras formas de nombrar", donde, o por un lado, se modifica el parentesco real, por otro parentesco ficticio, el cual, se identifica con el parentesco "practicado" o parentesco práctico mencionado por Bourdieu (2008) o, por otro lado, se crean formas nuevas o mixtas de nombrar a los parientes, sobre todo, a los parientes políticos. Respecto del primer ejemplo, encontramos que una de las mujeres que se define como "hija", llama a sus sobrinos-nietos "hijo" e "hija" ya que

abogase por los diminutivos empleados con oportunidad. (1904: 696-7) http://www.anales.uchile.cl/ index.php/ANUC/article/viewFile/24182/25520

${ }^{22}$ En relación con ello el trabajo de Schenider y Homans (1955) señala que cuando los padres se refieren a sus hijos con su primer nombre, no implica que la relación esté libre de las consideraciones de parentesco, es decir, de las obligaciones que el hijo tiene con su padre o el padre con su hijo.

${ }^{23}$ La formación de los hipocorísticos ha sido tratada por autores como Boyd-Bowman (1955), Urawa (1985), Báez (2002), entre otros. 
ejerce funciones maternales con ellos, puesto que su hermana, la madre de sus sobrinos, falleció. El siguiente relato denota este vínculo estrecho:

Mis sobrinos los que dejó mi hermana mayor que hace dos años que falleció son como mis hijos, son los que yo me preocupo de ellos ahora. A ellos les digo hijos. ("Mujer_27_2013, forma familiar unipersonal")

O también el siguiente relato donde encontramos el caso en el que como los primos son muy mayores, el entrevistado les llama "tíos", y a los hijos de sus primos, les llama "primos", aunque serían sus sobrinos según el término de referencia que le corresponde utilizar a este parentesco.

Bueno, no son parte como de mi familia nuclear, no tomo decisiones con ellos ni comparto ciertas cosas con ellos. Pero los veo regularmente y son muy cercanos, entonces, no sé en qué... en qué parte de... de esa extensión de familia corresponderían, no sé, por ejemplo, tengo, va a sonar extraño, son... en verdad son mis primos, pero son tan mayores que yo les digo "tíos". Entonces, los hijos de ellos que vendrían siendo mis sobrinos, yo les digo "primos". Y ellos vivían en la antigua casa, al lado, donde yo vivía en San Miguel. (Hombre_11_2013, Forma familiar monoparental extensa")

También el siguiente relato donde la hija se siente "mama" de su propia madre producto del trabajo de cuidado que ejerce sobre ella.

Yo siento que me hice cargo de mi mamá. Tan así que una vez que fuimos al hospital, en un episodio de puesta de marcapasos, que fue en el año dos mil doce, el año pasado, eh... me preguntaron en el mesón, a la entrada, pasó algo tan divertido, porque el señor que toma los datos a la entrada del hospital, ya, qué sé yo, viene, qué sé yo, "¿qué es usted de la, de la paciente?", jajá. Sí, y dije mamá. y fue como tan cómico, y nos miramos con la Ale y nos reímos, y la Ale dijo "Sí, es como la mamá, es como la mamá". Pero, no es que yo sea, tampoco me he sentido nunca la mamá así como que yo la mandaba, pero me hice cargo de ella completamente, me hice... y siento que lo hice de todo corazón, y todo, y sí, completamente. O sea, completamente, como pude haberme hecho cargo de una hija. (Mujer_3_2013, forma familiar extensa biparental")

Respecto al segundo ejemplo mencionado, nos encontramos con una familia recompuesta, donde la mujer entrevistada, llama a la pareja de su madre eesposo de mi madre" como término de referencia, y "tío" o "Tommy" como término de apelación. También explica cómo es su relación con su padre.

El esposo de mi mamá, que se llama Tom, le digo tío o Tommy de, de cariño. Conozco a Tom desde los ocho años. Entonces él en verdad es como mi segundo papá, él me crió en el fondo. (Mujer_23_2013, forma familiar ensamblada")

Mira, lo que pasa es que yo con mi papá, la relación con mi papá es muy especial, como que cortamos el vínculo hace un par de años por el tema de la carrera, entonces es como, como que mantenemos una relación como por, no sé cómo decirlo, como por cortesía en el fondo. Yo no llamo casi nada, entonces yo sé que él es de mi familia, entonces no sé si incluirlo ahí, porque yo sé que es de mi familia, pero no tengo como un vínculo... ("Mujer_23_2013, forma familiar ensamblada")

La misma entrevistada la cual se identifican como hija, llama "otras hermanas" a las hermanas que tiene por parte de padre, precisando el término de apelación a partir de los nombres personales, pero también habla de las hermanas que tiene por parte de la pareja actual de su madre. Refiere como "media hermana" a la hija del esposo de su madre y la llama "Ale": 
también incluía a mi media hermana, que es de la familia de mi papá, se llama Alejandra... ("Mujer_23_2013, forma familiar ensamblada")

O sea, es que, ay, es como tan rara mi relación, porque también por ejemplo la, la familia del esposo de mi mamá, yo con sus hijos viví cuando chica. Ellos viven lejos ya, también son como seudo parte de mi familia, por eso es como... («Mujer_23_2013, forma familiar ensamblada")

En resumen, nos encontramos con diminutivos, hipocorísticos y otras formas de nombrar que de alguna manera reflejan el tipo de relación que se tiene, o que se quiere intencionar entre aquellos considerados parientes.

\section{CONCLUSIONES}

Si bien analizar los términos de parentesco en nuestro trabajo parte de una gran diversidad de formas familiares, lo que hemos tratado de realizar es un primer acercamiento a las formas de nombrar a través de la categoría "trabajo de parentesco". Al tomar ese concepto "trabajo de parentesco" como punto de partida tratamos de recoger la dinámica misma del trabajo del mantenimiento de los vínculos aproximándonos a las formas de nombrar que resultan de él. Mostramos a través de los relatos de los entrevistados/as que más allá de la forma familiar preexistente, nombrar, es una forma de practicar las posiciones de género y parentesco al interior de las familias, las cuales, no se encuentran alejadas en ningún momento de ciertas jerarquías y relaciones de poder. Así pues, para aplicar esta concepción dinámica del trabajo de parentesco hemos partido de las formas familiares operantes en el momento dado de cada entrevista realizada para desde allí acudir a las prácticas efectivas que lo producen, las condiciones sociales que las provocan y mantienen y, sobre todo, los términos de parentesco que se usan para mantener esas relaciones. Por medio de la carta de parentesco se ha tratado de visibilizar las posiciones de parentesco con los que se identifican los entrevistados/as y las responsabilidades derivadas de estos, las cuales están asociadas a una determinada forma de nombrar en la que predominan los diminutivos, los hipocorísticos, y "otras formas de nombrar". Producto de lo mencionado nos damos cuenta que en el trabajo de mantenimiento de los vínculos las mujeres, identificadas como madres, hijas, hermanas, o amigas, manipulan las formas de nombrar, ya sea por medio de los dimuntivos, los hipocorísticos, u otras formas, producto del trabajo de mantenimiento de los vínculos relacionados con el cuidado que realizan con esas personas o del cuidado que reciben de sus parientes. Aunque mostrar esta relación entre trabajo de parentesco, relaciones de género y poder y formas de nombrar no es fácil, este artículo constituye una primera aproximación para seguir afinando que "la terminología de parentesco es uno de los aspectos en donde se visibilizan las disonancias, contradicciones y vacíos que genera la tensión entre lo biológico y lo social en la construcción del parentesco, como resultado de los plurales modos de "vivir en familia" que practican los sujetos" (Rivas y Moncó 2007:12). 


\section{BIBLIOGRAFÍA CITADA}

Alberdi, Inés. 1999. La nueva familia española. Madrid: Taurus.

Amunátegui, Miguel. 1904. "Una lección sobre diminutivos". Anales de la Universidad de Chile: 695718. doi. 10.5354/0717-8883.1904.24182.

Arriagada, Irma (ed.). 2008. Futuro de las familias y desafíos para las políticas públicas. Santiago: CEPAL.

Baéz, Gloria E. 2002. "Vitalidad y tradición de hipocorísticos empleados en la ciudad de México: 1955-1999. Un estudio comparativo". Anuario de Letras, XL: 241-265.

Bello, Andrés. 1984. Gramática de la lengua castellana. Madrid: Editorial EDAF.

Bestard, Joan. 2009. "Los hechos de la reproducción asistida: entre el esencialismo biológico y el constructivismo social. Revista de antropología social, 18: 83-95.

Boyd-Bowman, Peter. 1955. "Cómo obra la fonética infantial en la formación de los hipocorísticos". Nueva Revista de Filología Hispánica, 9 (4): 337-366.

Bourdieu, Pierre. 2008. El sentido práctico. Madrid: Siglo XXI.

Cadoret, Anne. 2003. Padres como los demás: homosexualidad y parentesco. Barcelona: Gedisa.

Callebaut, Sien. 2011. "Entre sistematización y variación: el sufijo diminutivo en España y en Hispanoamérica. Disponible en: http://lib.ugent.be/fulltxt/RUG01/001/786/647/RUG01-001786647_ 2012_0001_AC.pdf. Fecha de acceso: 15 de junio de 2015.

Carrington, Christopher. 1999. No Place Like Home: Relationships and Family Life among Lesbian and Gay Men. Chicago: University of Chicago Press.

Carsten, Janet. 2004. After kinship. Cambridge: Cambridge University Press.

Cicerchia, Ricardo. 1999. "Alianzas, redes y estrategias. El encanto y la crisis de las formas familiares". Nómadas, 11: 46-53.

Encuesta de Caracterización Socioeconómica (CASEN). 2013. Santiago: MIDEPLAN.

Collier, Jane y Sylvia Yanagisako (eds.). 1987. Gender and Kinship. Essays toward a unified analysis. California: Stanford University Press.

Comas d'Argemir, Dolors. 2010. "Cartas de parentesco y genealogías", en Joan Pujadas, Dolors Comas d'Argemir y Jordi Roca (eds.), Etnografía: 135-142. Barcelona: Editorial UOC.

Del Valle, Teresa. 2010. "La articulación del parentesco y el género desde la antropología feminista", en Virginia Fons, Anna Piella y María Valdés (eds.), Procreación, crianza y género. Aproximaciones antropológicas a la parentalidad: 295-318. Barcelona: PPU.

Di Leonardo, Micaela. 1987. "The Female World of Cards and Holidays: Women, Families, and the Work of Kinship". Signs (12) 3: 440-453.

González, María del Mar. 2009. "Nuevas familias, nuevos retos para la investigación y la educación”. Cultura y Educación 21 (4), 381-389.

Gonzálvez, Herminia. 2013. "La producción científica sobre la familia en Chile: miradas desde la antropología feminista". Revista de Estudios de Género. La ventana, 4 (38): 88-119.

Gonzálvez, Herminia. 2015. Diversidades familiares, cuidados y migración. Nuevos enfoques y viejos dilemas. Santiago de Chile: Editorial Alberto Hurtado.

Gonzálvez, Herminia. 2016. "El "trabajo de parentesco" que realizan las familias en Santiago de Chile". Revista de antropología social, 25-1: 153-169. doi: 10.5209/rev_RASO.2016.v25.n1.52629.

Gonzálvez, Herminia. En prensa. "Las familias transnacionales ¿una tautología? Más allá de la dicotomía proximidad/distancia”. Revista Polis (aceptado).

Grau, Jordi. 2006. Procreación, género e identidad. Debates actuales sobre el aprentesco y la familia en clave transcultural. Barcelona: Ediciones Bellaterra.

Gregorio, Carmen. 2006. "Contribuciones feministas a problemas epistemológicos de la disciplina antropolófica: representación y relaciones de poder". AIBR. Revista de Antropologia Iberoamericana. 1(1): 22-39.

Gregorio, Carmen. 2011. "Análisis de las migraciones transnacionales en el contexto español, revisitando la categoría género desde una perspectiva etnográfica y feminista". Nueva Antropologia, XXIV 74: 39-7.

Gregorio, Carmen y Herminia Gonzálvez. 2012. "Las articulaciones entre género y parentesco en el contexto migratorio: más allá de la maternidad transnacional." Ankulegi 16: 43-17. 
INE (2015). Chile. Proyecciones y estimaciones de población. País y regiones. Santiago de Chile: Instituto Nacional de Estadística

Jelin, Elisabeth. 1998. Pan y afectos. La transformación de las familias. Buenos Aires: Fondo de Cultura Económica.

Jociles, María Isabel y Ana María Rivas. 2010. “Motivaciones genéticas y experienciales: El discurso de las MSPE sobre la fecundación asistida como vía de acceso a la maternidad en solitario". Alteridades 20(39): 107-124.

Jociles, María Isabel y Fernando Villaamil. 2012. "Madres solteras por elección: representaciones sobre la fecundación sexual como vía de acceso a la maternidad". Chungará 44(4): 717-731.

Jociles, Maria Isabel y Raquel Medina. 2013. La monoparentalidad por elección. El proceso de construcción de un modelo de familia. Valencia: Tirant lo Blanch.

Konvalinka, Nancy (ed.). 2012. Modos y maneras de hacer familia. Las familias tardias, una modalidad emergente. Madrid: Biblioteca Nueva.

Lamphere, Louise. 1987. "Feminism and Anthropology. The struggle to reshape our thinking about gender" en Christie Farbham (ed.) The Impact of Feminist Research in the Academy: 11-33. Bloomington: Indiana University Press.

León, Magdalena. 1994. "¿La identidad se construye en la familia?" En Rodríguez, Regina. (ed.), Familias siglo XXI: 29-42. Santiago: ISIS International.

Needham, Rodney (ed.). 1971. Rethinking Kinship and Marriage. Londres: Tavistock.

Moncó, Beatriz y Ana María Rivas. 2007. "La importancia de 'nombrar'. El uso de la terminología de parentesco en las familias reconstituidas". Gazeta de Antropología 23: 1-14.

Morera, Marcial (2006). Diminutivos, apodos, hipocorísticos, nombres de parentesco y nombres de edad en el sistema de tratamientos populares de Fuerteventura. En: file:///C:/Users/hgonzalv/ Downloads/Dialnet-DiminutivosApodosHipocoristicosNombresDeParentesco-2234105.pdf

Morgan, Lewis Henry. 1871. Sistemas de consanguinidad y afinidad de la familia humana.

Olavarría, José. 2014. "Transformaciones de la familia conyugal en Chile en el período de la transición democrática (1990-2011)". Revista Polis n. ${ }^{\circ}$ 37: 1-19.

Palerm, Angel. 1997. Introducción a la teoría etnológica. México: Universidad Iberomericana.

Peletz, Michael. 1995. "Kinship studies in late twentieth-century anthropology". Annual Review of Anthropology: 343-368.

Rivas, Ana María. 2007. "Transformaciones socioculturales y cambios familiares: continuidades y rupturas", en Carmelo Lisón (ed.), Introducción a la antropología social y cultural. Teoría, método y práctica: 105-128. Madrid: Akal.

Rivas, Ana María. 2009. "Pluriparentalidades y parentescos electivos". Revista de Antropología Social 18: 7-19.

Robichaux, David (Comp.). 2007. Familia y Diversidad en América Latina. Estudios de casos. Buenos Aires: CLACSO.

Rotenberg, Eva y Beatriz Agrest Wainer (Comp.) 2007. Homoparentalidades. Nuevas familias. Buenos Aires: Lugar Editorial.

Salvo, Irene. 2015. Narrativas de mujeres que adoptaron individualmente en Chile: Tensiones, desafios y perspectivas (Tesis doctoral inédita). Facultad de Psicología, Universidad de Buenos Aires, República Argentina.

Salvo, Irene y Herminia Gonzálvez. 2015. "Monoparentalidades electivas en Chile: Emergencias, tensiones y perspectivas". Psicoperspectivas. Individuo y sociedad 14 (2): 40-50.

Sanz, Jesús; María José Pont; Consuelo Álvarez; Herminia Gonzálvez; María Isabel Jociles; Nancy Konvalinka; José Ignacio Pichardo; Ana María Rivas y Elisa Romero. 2013. "Diversidad familiar: Apuntes desde la antropología social”. Revista de treball social n. ${ }^{\circ}$ 198: 30-40.

Scheffler, Harold. 1991. "Sexism and naturalism in the study of kinship", en Micaela Di Leonardo (ed.), Gender at the crossroads of knowledge. Feminist anthropology in the postmodern era: 361381. California: University of California Press Books.

Schneider, David. 1968. American Kinship: A cultural account. Chicago: University of Chicago Press. Schneider, David. 1984. A critique of the Study of Kinship. Ann Arbor: University of Michigan Press. Schneider, David y George C. Homans. 1955. "Kinship terminology and the American Kinship system" American anthropologist. 57(6):1194-1208. 
Segalen, Martine. 1992. Antropología histórica de la familia. Madrid: Taurus.

Segalen, Martine. 2007. "El parentesco en la antropología actual: De las sociedades "exóticas" a las sociedades "modernas"', en David Robichaux (Comp.) Familias Mexicanas en Transición. Unas miradas antropológicas: 39-60. México: Universidad Iberoamericana.

Tasker, Fiona. 2005. "Lesbian mothers, gay fathers, and their children: a review". Developmental and Behavioral Pediatrics 26 (3): 224-240.

Tubert, Silvia. 2001. Deseo y representación. Convergencias de psicoanálisis y teoría feminista. Madrid: Síntesis.

Uraba, Mikío. 1985. "Muestra de hipocorísticos en el español bogotano". Thesaurus, 40: 51-102.

Yanagisako, Sylvia. 1979. "Family and household: the analysis of domestic groups". Annual review of anthropology 8: 161-205.

Yanagisako, Sylvia y Carol Delaney. 1995. "Naturalizing Power», en Sylvia Yanagisako y Carol Delaney (eds.) Naturalizing Power. Essays in Feminist Cultural Analysis: 1-21. New York-London: Routledge.

Valenzuela, Samuel; Eugenio Tironi y Timothy Scully (eds.). 2006. El eslabón perdido. Familia, modernización y bienestar en Chile. Santiago: Taurus.

Zuluaga, Alberto. 1970. "La función del diminutivo en español", Thesaurus: Boletín del Instituto Caro y Cuervo 25(1): 23-48.

Fecha de recepción: 4 de agosto de 2015

Fecha de aprobación: 21 de enero de 2016 